\section{AVALIAÇÃO DO RISCO PARA DIABETES PELA ANÁLISE DA ÍRIS: UMA POSSIBILIDADE*}

\section{EVALUATION OF THE RISK FOR DIABETES THROUGH THE ANALYSIS OF IRIS: A POSSIBILITY}

\section{RESUMO}

Diabetes é um problema de saúde pública. Métodos que identifiquem precocemente a predisposição para a doença devem ser investigados. Iridologistas afirmam que o Sinal do Pâncreas e a Cruz de Andréas sugerem predisposição para diabetes. Objetivos: Verificar a prevalência do sinal do Pâncreas e da Cruz de Andréas em indivíduos com e sem a diabetes e a correlação com a taxa de glicemia. Métodos: A coleta de dados ocorreu entre fevereiro de 2010 e junho de 2011. Participaram 410 indivíduos com idade superior a 30 anos. Resultados: Indivíduos com diabetes apresentaram maior prevalência dos sinais iridológicos estudados. $O$ teste $t$ de Student aponta diferença estatisticamente significativa na prevalência desses sinais entre pacientes com e sem diabetes e entre indivíduos com e sem antecedentes familiares para a doença. A análise do coeficiente de correlação de Pearson mostra correlação entre estes sinais e a glicemia alterada. Conclusão: Concluímos que esses sinais sugerem predisposição para Diabetes.

\section{PALAVRAS-CHAVE}

Diabetes Mellitus (prevenção).

Iridologia.

Enfermagem.

Medicina integrativa.
Léia Fortes Salles

- Enfermeira pela Escola de Enfermagem da Universidade de São Paulo.

Doutora em Ciências da Saúde pelo Programa da Saúde do Adulto da EEUSP. Especialista em Iridologia e Irisdiagnose e em Terapia Floral. Membro do Grupo de Estudos em

Práticas Complementares em Saúde do Conselho Nacional de Desenvolvimento Científico e Tecnológico (CNPq).

Autor responsável para

correspondência.

E-mail:leia.salles@usp.br

Maria Júlia Paes da Silva

- Enfermeira. Professora Titular do

Departamento Médico-cirúrgico da

Escola de Enfermagem da Universidade de São Paulo. Líder do Grupo de

Estudos em Práticas Alternativas e Complementares em Saúde do Conselho Nacional de Desenvolvimento Científico e Tecnológico (CNPq). E-mail:juliaps@usp.br.

Celso Fernandes Battello

- Médico, mestre em Homeopatia pela Faculdade de Ciências de São Paulo

e Instituto Brasileiro de Estudos

Homeopáticos (FACIS-IBEHE).

Email:battello@battello.med.br.

*Dados retirados da tese apresentada à Escola de Enfermagem da Universidade de São Paulo intitulada "Avaliação da prevalência e da herdabilidade dos sinais iridológicos que sugerem predisposição para diabetes em indivíduos com e sem a doença" em 2012.

CORRESP ONDENT E

Léia Fortes Salles

Endereço: Rua Tucuna, $n^{\circ}$ 742, apto 132. Pompéia

E-mail:e-mail:leia.salles@usp.br

Recebido: 04/10/13

Aprovado: 23/12/13 


\begin{abstract}
The method to identify early the predisposition for Diabetes mellitus should be investigated, since the disease is a public health problem. Scholars of iridology claim that the Sign of Pancreas and the Cross of Andreas suggest predisposition to diabetes. Objectives: To determine the prevalence of the Sign of Pancreas and the Cross of Andreas in subjects with and without the disease and the correlation with the rate of glucose. Methods: Data collection occurred between February 2010 and June 2011. Participants 410 individuals older than 30 years treated at the Health Center School. Results: Individuals with diabetes had a higher prevalence of signs studied iridology. The Student $t$ test showed statistically significant differences in the prevalence of these signs between patients with and without diabetes and among individuals with and without family history of the disease. The Pearson Correlation Coefficient shows the correlation of iridology signs studied and blood glucose alteration. Conclusion: We conclude that these signs suggest a predisposition to diabetes.
\end{abstract}

KEY WORDS: Diabetes Mellitus (prevention). Iridology. Nursing. Integrative Medicine.

\section{INTRODUÇÃO}

O crescimento das doenças crônicos degenerativas deve-se, em parte, ao rápido envelhecimento da população. Um dos desafios mundiais de saúde é encontrar novos e mais eficazes modos de prevenir o aparecimento de doenças crônico-degenerativas e suas incapacidades, como por exemplo do Diabetes Mellitus ${ }^{(1)}$.

O Diabetes Mellitus (DM) destaca-se pela sua incidência e prevalência, além dos altos índices de morbidade e mortalidade relacionados à enfermidade e às suas complicações ${ }^{(2)}$. Segundo a Organização Mundial de Saúde mais de 220 milhões de pessoas têm diabetes e este número deve duplicar até $2030^{(3)}$.

A doença caracteriza-se pela resistência à insulina e baixa secreção do hormônio, tem componente genético, porém está atrelada à obesidade, sedentarismo e a outros fatores ambientais ${ }^{(4)}$.

Dentre as profissões da área da saúde, a Enfermagem foi pioneira no pesquisa, por meio da Resolução do Conselho Federal de Enfermagem (COFEN) 197/1997 (19/01/1997) $)^{(5-6)}$.

As informações fornecidas pela íris podem ser utilizadas durante todo o processo de Enfermagem, contribuíndo para uma assistência integral ${ }^{(7)}$. Portanto, neste estudo investigamos se por meio da análise da íris é possível detectar a predisposição para Diabetes Mellitus.
Irisdiagnose é uma ciência que permite identificar por meio da íris aspectos físicos, emocionais e mentais do indivíduo ${ }^{(8)}$. É um método propedêutico que ajuda conhecer, em um dano momento, órgãos debilitados no organismo e seus estágios evolutivos. Seu objetivo é detectar distúrbios em evolução e intervir precocemente para evitar o adoecimento ${ }^{(8)}$.

Fundamentando a Iridologia, temos que a íris é uma extensão do cérebro, conectada com todos os órgãos e tecidos do corpo, via tálamo óptico e sistema nervoso, revelando a condição das áreas mais remotas do organismo, por meio das mudanças nas fibras da íris ${ }^{(9)}$.

O Sinal do Pâncreas e a Cruz de Andréas são dois sinais na íris que os profissionais que utilizam a iridologia afirmam sugerir predisposição para o diabetes $^{(8-10)}$.

O Sinal do Pâncreas indica que o pâncreas é um órgão de choque, aquele que sofreu alterações no seu desenvolvimento embriológico e tornou-se debilitado e que frente a estímulos nocivos, poderá adoecer. No mapa condensado da iridologia, o pâncreas está localizado na íris direita às 7 horas (comparando-se a íris a um relógio). Este sinal aparece como abertura das fibras da íris indicando diminuição da densidade do tecido (Figura 1). 

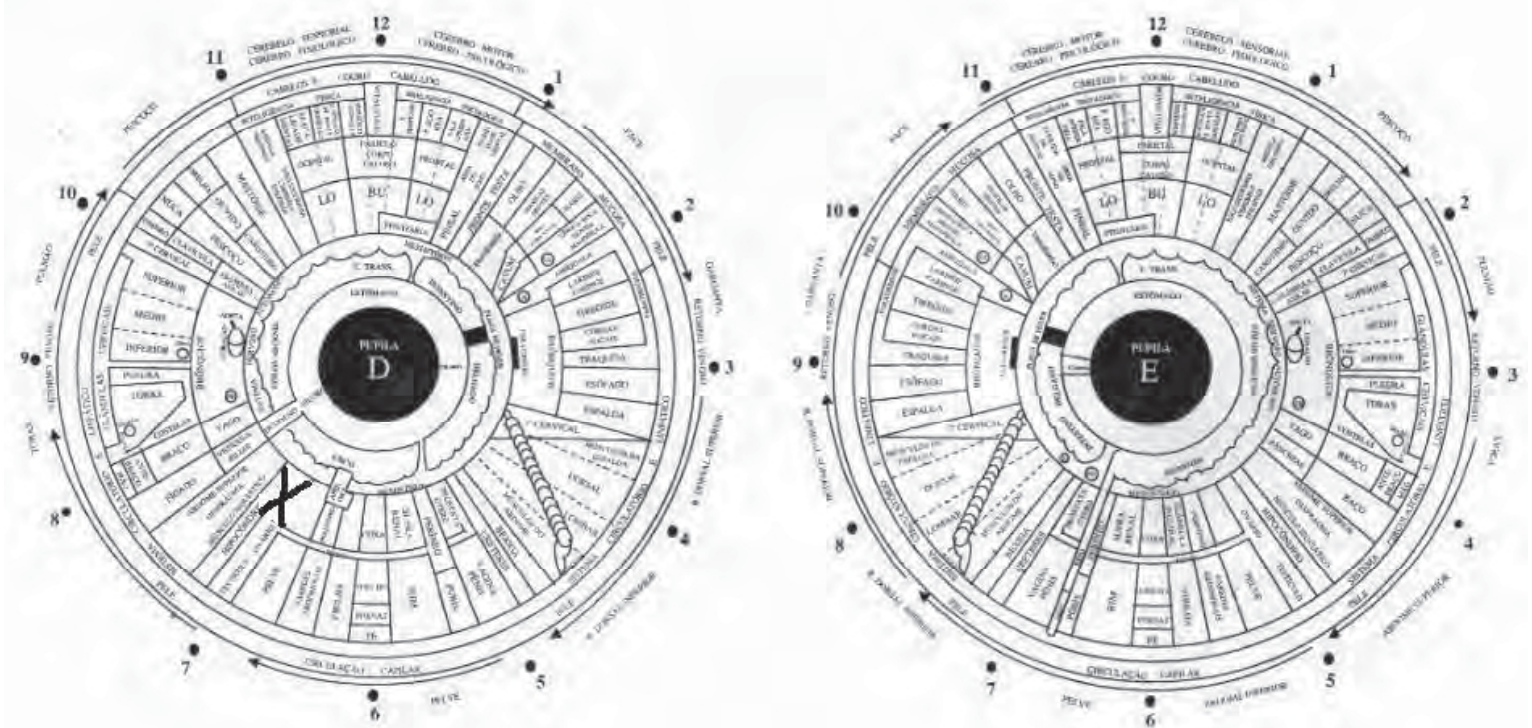

Figura 1: Mapa condensado da iridologia e sinal do pâncreas.

Fonte: Battello CF. Iridologia e irisdiagnose: o que os olhos podem revelar. Ed. Ground; 2009(8).

O outro sinal sugerido pela iridologia é a Cruz de Andréas. Este sinal indica especificamente predisposição para diabetes, representando disfunção endócrina e exócrina do pâncreas. Ele pode ser visualizada em am-

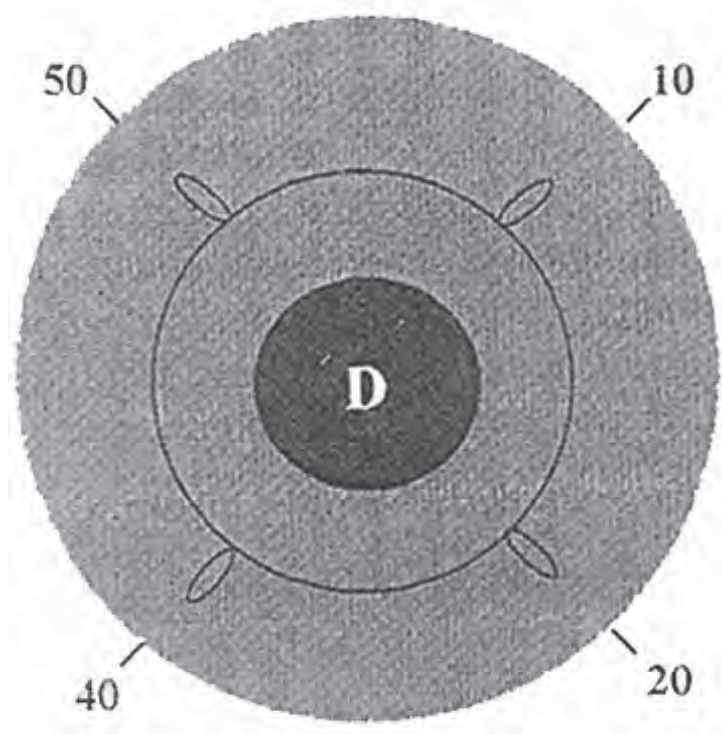

bos os olhos em forma de quatro lacunas (aberturas das fibras) dispostas as 10, 20, 40 e 50 minutos, comparando-se a íris com o relógio (Figura 2). Estas lacunas significam tecidos menos densos, predispostos a adoecer.

Figura 2: Cruz de Andréas.

Fonte: Battello CF. Iridiologia total. Ed. Ground; 1996.p. 52 $2^{(10)}$.

São pouquíssimas as pesquisas encontradas sobre o assunto, apesar dos benefícios que esta proposta pode trazer na presença dos sinais. Um estudo realizado em São Paulo (BR), com 97 indivíduos portadores de diabetes, encontra uma prevalência de $98 \%$ do sinal do pâncreas e $89 \%$ da Cruz de Andréas nestes pacientes e correlaciona estes sinais

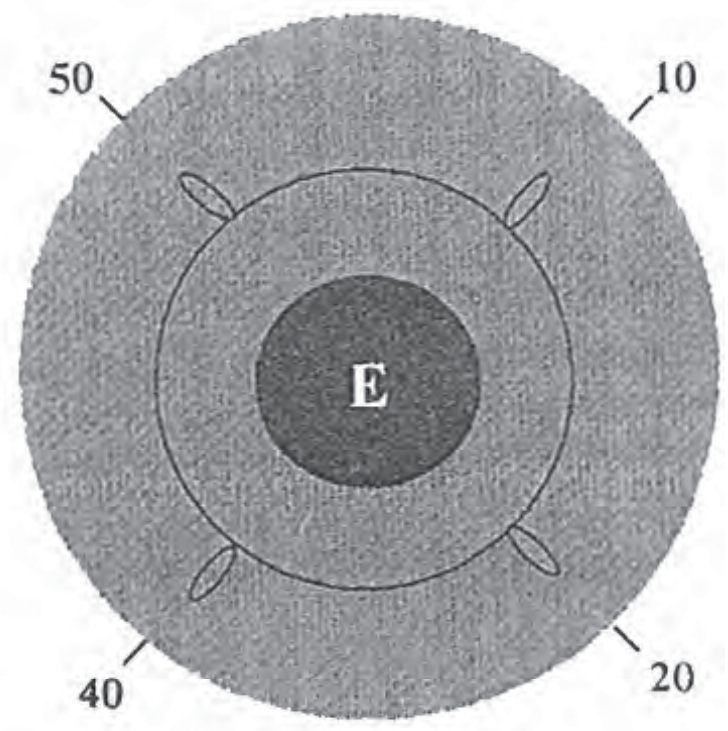

com antecedentes familiares para a doença, sedentarismo e obesidade ${ }^{(7)}$.

Em outra investigação, indivíduos com e sem diabetes são comparados e é encontrada diferença estatisticamente significativa entre os grupos em relação a ambos os sinais iridológicos que sugerem predisposição para Diabetes Mellitus ${ }^{(11)}$. 
Estudo duplo cego, realizado no Município de Entre-Ijuis, detecta 75\% de correspondência entre os sinais iridológicos sugestivos da predisposição de DM e exames laboratoriais que auxiliam no diagnóstico da doença ${ }^{(12)}$.

As investigações apresentados estudam os sinais que sugerem predisposição para Diabetes Mellitus somente em indivíduos com a doença. O presente estudo pretende comparar a prevalência do Sinal do Pâncreas e Cruz de Andréas, em indivíduos com e sem diabetes e verificar a correlação entre os sinais, a doença e a taxa da glicemia.

A relevância do estudo baseia-se nas estatísticas sobre Diabetes Mellitus, que reforçam a magnitude do problema e a urgência de colocar em prática medidas de prevenção para evitar o surgimento da doença e de suas complicações. Para não desenvolver o Diabetes Mellitus, a alimentação e a atividade física são importantes e podem minimizar os efeitos da carga genética favorável ao DM.

Por outro lado, hábitos iniciados na infância são mais fáceis de serem mantidos. Assim sendo, a proposta da Iridologia de detectar precocemente a predisposição para o DM parece muito interessante por permitir o início dos ajustes no comportamento alimentar e em relação à atividade física em idades tenras, facilitando a manutenção de hábitos adequados para evitar o desenvolvimento da doença e suas graves complicações.

\section{MÉTODO}

Trata-se de um estudo exploratório, descritivo, de campo, transversal, com abordagem quantitativa e duplo cego ${ }^{(13)}$.

A pesquisa foi realizada no Centro de Saúde-Escola "Geraldo de Paula Souza", na cidade de São Paulo, no período de Fevereiro de 2010 a Junho de 2011, após aprovação do Comitê de Ética e Pesquisa da Escola de Enfermagem da USP (Processo nº63/2009/ CEP-EEUSP).

Participaram do estudo 410 indivíduos após concordância e assinatura do Termo de Consentimento Livre e Esclarecido divididos em três grupos - G1: com Diabetes Mellitus (DM), G2: sem diabetes e com antecedentes familiares para a doença (sem DM e com AFDM), G3: sem diabetes e sem antecedentes familiares para a doença (sem DM e sem AFDM).

Os critérios de inclusão foram: estar inscrito no Centro de Saúde Escola "Geraldo de Paula Souza", exceto para os familiares; ter idade superior a 30 anos e; saber sobre sua antecedência pessoal em relação ao DM.

Os critérios de exclusão foram dificuldade em abrir e manter os olhos abertos; pterígios que encobrisse mais do que um quadrante do olho; imagens sem foco e; voluntários que não coletaram o exame de glicemia de jejum.

Os dados foram coletados por meio da identificação do sujeito, preenchimento da ficha clínica e fotografia de ambas as íris pela autora especialista em Iridologia. A captação das imagens de ambas as íris foi realizada com o auxílio de uma câmara digital acoplada a uma lente iridológica com luminosidade adequada. As fotos foram gravadas em memory card.

Os exames de glicemia foram xerocopiados do prontuário do paciente quando o resultado era recente (menos de três meses da entrevista) ou solicitado novo exame pela pesquisadora.

A análise da íris foi realizada por um médico da Associação Brasileira de Iridologia, que não teve acesso aos dados dos pacientes. Foi - lhe entregue um memory card com os arquivos de cada participante, contendo imagens de ambas as íris.

Os dados foram tratados estatisticamente. Adotou-se nível de significância $=0,05$ e intervalo de confiança de $95 \%$.

\section{RESULTADOS}

Foram recrutados 410 pacientes e incluídos no estudo 356, que atenderam aos critérios de inclusão. Os motivos para exclusão foram a não realização do exame de glicemia de jejum (29,7\%), a falta de foco na imagem $(64,8 \%)$ e a presença de pterígeo ou cicatriz encobrindo mais de um quadrante da íris $(5,5 \%)$.

Após análise dos dados obtivemos a caracterização demográfica da amostra de 356 pacientes que participaram da pesquisa, apresentada a seguir. 
A idade dos pacientes variou entre 30 e 91 anos, com média de 55,2 anos. Quanto ao gênero, 265 $(74,5 \%)$ indivíduos eram do sexo feminino e 91 $(25,5 \%)$ do sexo masculino.

Com base na classificação do Índice de Massa Corpórea (IMC) ${ }^{(14)}$, proposta pela OMS, nos três grupos observa-se o sobrepeso. O G3 se destaca pela incidência de indivíduos com pré-obesidade $(47,4 \%)$, os G1 e G2 pelas taxas semelhantes de obesidade (27\%).

Conforme a recomendação americana sobre atividade física ${ }^{(15)}$, o sedentarismo foi prevalente nos três grupos, sendo maior no G3 (69,5\%), seguidos do G2 (66,7\%) e G1 (63,6\%).

No G1, formado por indivíduos portadores de diabetes, $87,5 \%$ referiu ter antecedente familiar para a doença.

Quanto à glicemia, as taxas foram mais alteradas no G1 (91,6\%), seguidos pelo G2 (57,2\%) e G3 $(11,6 \%)$. Dentro do período de coleta de dados, no G2 11 pessoas foram diagnosticadas com Diabetes Mellitus e passaram a fazer uso de medicamento para a doença. Neste mesmo período, nenhum voluntário do G3 foi diagnosticado com a doença.

A prevalência do Sinal do Pâncreas e da Cruz de Andréas foi de $100 \%$ e $87,5 \%$ no G1, 99,4\% e 90,3\% no G2 e, 55,8\% e 25,3\% no G3; respectivamente.

Tabela 1: Comparação do Sinal do Pâncreas e Cruz de Andréas nos três grupos. São Paulo, 2011.

\begin{tabular}{|c|c|c|c|}
\hline & G 1 & G 2 & G 3 \\
\hline \multicolumn{4}{|c|}{ SINAL DO PÂNCREAS } \\
\hline Sim & $96100 \%$ & $16499,4 \%$ & $5355,8 \%$ \\
\hline Não & $00 \%$ & $010,6 \%$ & $4244,2 \%$ \\
\hline \multicolumn{4}{|c|}{ CRUZ DE ANDRÉAS } \\
\hline Sim & $8487,5 \%$ & $14990,3 \%$ & $2425,3 \%$ \\
\hline Não & $1212,5 \%$ & $169,7 \%$ & $7174,7 \%$ \\
\hline
\end{tabular}

Legenda: G1 (DM), G2 (sem DM e com AFDM), G3 (sem DM e sem AFDM).

O teste $t$ de Student apontou diferença entre os grupos com diabetes (G1) e sem diabetes (G2 + G3) em relação a prevalência da Sinal do Pâncreas $[\mathrm{t}(356)=-7,264 ; \mathrm{p}=0,000]$ e Cruz de Andréas $[\mathrm{t}(356)=-4,754 ; \mathrm{p}=0,000]$.
Os G2 e G3 são formados por indivíduos sem Diabetes Mellitus. $\mathrm{O}$ que diferencia esses grupos foi a antecedência familiar para a doença. $O$ teste $t$ de Student apontou diferença entre os grupos de pacientes com antecedentes para diabetes (G2) e sem antecedentes para diabetes (G3) em relação a prevalência do Sinal do Pâncreas $[\mathrm{t}(260)=8,638 \mathrm{p}=0,000]$ e Cruz de Andréas $[\mathrm{t}(260)=13,256 ; \mathrm{p}=0,000]$.

Por último, foi comparando apenas indivíduos com (G1) e sem a doença (G3). 0 teste $t$ de Student demonstrou diferença estatisticamente significativa entre as médias dos pacientes com Sinal do Pâncreas e Cruz de Andréas entre o grupo G1 e G3 (p=0,000).

O teste Chi Quadrado foi utilizado para saber se o sinais estudados podem influenciar no desenvolvimento do diabetes e foi constatado que ter a doença depende de ter ambos os sinais e, que somente a presença do Sinal de Pâncreas não é suficiente para desencadear a doença.

O coeficiente de correlação de Pearson apontou correlação entre a prevalência do Sinal do Pâncreas $(r=0,554)$ e alteração na glicemia $(r=0,376)$ e entre a prevalência da Cruz de Andréas e AFDM ( $\mathrm{r}=$ $0,602)$ e alteração na glicemia $(r=0,359)$.

\section{DISCUSSÃO}

No Brasil, a prevalência da obesidade baseada no IMC passou de $2,4 \%$ na década de 70 para $6,9 \%$, na década de $90^{(16)}$. Enquanto G1 e G2 apresentam mais obesidade, $\mathrm{G} 3$, grupo sem DM, apresenta pré-obesidade. Porém, as diferenças no IMC entre os três grupos não são significativas. Nos três grupos, a grande maioria foi de indivíduos matriculados e tratados no Centro de Saúde, onde uma das recomendações, tanto para portadores de DM, quanto para indivíduos com outras patologias, é o seguimento de dieta alimentar.

Entre os participantes da pesquisa destaca-se 0 sedentarismo e pode-se perceber uma maior preocupação nos indivíduos com diabetes, talvez pelo fato de serem orientados que uma das formas de controle da glicemia é a atividade física.

$\mathrm{Na}$ amostra estudada, $87,5 \%$ dos indivíduos com diabetes relatam antecedentes familiares para 
a doença. As pesquisas apontam que o diabetes tipo 2 tem forte componente genético e apresenta padrão de herança multigenética, embora os fatores ambientais, além do sedentarismo e da obesidade terem participação no desenvolvimento da doença ${ }^{(17-18)}$.

Mesmo estando em tratamento com hipoglicemiantes orais e/ou insulina, $91,6 \%$ do G1 estava com a glicemia alterada contra apenas $11,6 \%$ no G3. No G2, mais da metade $(57,6 \%)$ já têm sua glicemia alterada. Quando comparado com o G3 (11,6\%) fica clara a influência da hereditariedade no desenvolvimento do DM.

Em pesquisas anteriores ${ }^{(7,11)}$ foi demonstrado que ambos os sinais iridológicos tinham correlação com antecedentes familiares para a doença, sedentarismo e obesidade. No presente estudo, evidenciou-se correlação dos sinais apenas com antecedentes familiares e com a taxa de glicemia. Esta última variável não foi estudada no primeiro trabalho que investiga a prevalência dos sinais em indivíduos com diabetes $^{(7)}$.

Indivíduos com diabetes apresentaram maior prevalência dos sinais iridológicos estudados. O G1 e G2 tiveram índices semelhantes na prevalência dos sinais e maiores que o $\mathrm{G} 3$.

Conforme a literatura de iridologia ${ }^{(8-10)}$, o Sinal do Pâncreas não é específico para DM; apenas mostra que o pâncreas é um orgão sensível, ou seja, é delibitado e frente a estímulos nocivos está sujeito a inúmeras doenças. Nesta pesquisa identificamos que este sinal apareceu em todos os indivíduos com diabetes, em $99,4 \%$ do G2, e em $55,8 \%$ no G3.

Já o sinal da Cruz de Andréas, considerado específico para DM, apareceu em $87,5 \%$ dos portadores
DM contra 25,3\% do G3.

Não foram encontrados nas bases de dados estudos com estes sinais iridológicos comparando grupo com e sem a diabetes. Para os pacientes com diabetes, os dados deste estudo corroboram com os resultados anteriores ${ }^{(7,11-12)}$.

\section{CONSIDERAÇÕES FINAIS E CONCLUSÃO}

Indivíduos com diabetes apresentaram maior prevalência dos sinais iridológicos estudados. O G1 e G2 tiveram índices semelhantes na prevalência dos sinais e maiores que o G3.O teste $t$ de Student aponta diferença estatisticamente significativa na prevalência desses sinais entre pacientes com e sem diabetes e entre indivíduos com e sem antecedentes familiares para a doença. O Chi Quadrado demonstra que ter ambos os sinais aumenta a chance do desenvolvimento do diabetes. $O$ coeficiente de correlação de Pearson mostra correlação entre os sinais estudados e a taxa de glicemia alterada.

Diante dos pouquíssimos estudos sobre o tema e da possibilidade de especialização do enfermeiro no uso de uma "ferramenta" de análise do indivíduo, que possa auxiliá-lo a prevenir desequilíbrios ou doenças, os resultados desse estudo contribuem para a compreensão correta de sinais que sugerem pré-diagnose do DM.

A análise da íris na infância permite ao profissional de saúde, devidamente capacitado, tomar medidas cabíveis para evitar doenças futuras incentivando, neste caso, a alimentação adequada e a atividade física.

Concluímos que o Sinal do Pâncreas e a Cruz de Andréas sugerem predisposição para Diabetes Mellitus.

\section{CONFLITO DE INTERESSES}

Declararam não haver

\section{FONTE DE FINANCIAMENTO}

Não se aplica 


\section{REFERÊNCIAS}

1. OMS. Cuidados inovadores para condições crônicas: componentes estruturais de ação. Brasília; 2003.

2. Brasil. A vigilância, o controlo e a prevenção das doenças crônicas não-transmissíveis: DCNT no contexto do Sistema Único de Saúde brasileiro. Brasília: Ministério da Saúde - Organização PanAmericana da Saúde, 2005.

3. World Health Organization (WHO). Diabetes - Key facts [online]. Available from: http: // www.who.int/mediacentre/factsheets/fs312/ en/index.html. [Acesso em 13 jun. 2011].

4. Gross JL, Silveiro SP, Camargo JL, Reichelt AJ, Azevedo MJ. Diabetes Mellitus: Diagnosis, classification and glucose control evaluation. Arq Bras Endocrinol Metab. 2002; 46(1):16-26.

5. Salles LF, Silva MJP. Práticas Complementares na Assistência de Enfermagem aos Adultos. In: Associação Brasileira de Enfermagem. Programa de Atualização em Enfermagem - Saúde de Adulto (PROENF). Porto Alegre: Artmed; 2009. Ciclo 4, módulo 1, p. 73 107.

6. Conselho Federal de Enfermagem (COFEN). Resolução COFEN -197/1997. Estabelece e reconhece as Terapias Alternativas como especialidade e/ ou qualificação do profissional de Enfermagem In: Conselho Regional de Enfermagem de São Paulo (COREnSP). Documentos básicos de enfermagem: enfermeiros, técnicos, auxiliares. São Paulo; 2001.p. 159 - 60.

7. Salles LF, Silva MJP, Araújo EAC. Prevalence of iridologic signs in individuals with Diabetes mellitus. Acta paul. Enferm. 2008; 21(3):474-80.

8. Batello CF. Iridologia e irisdiagnose: o que os olhos podem revelar. $3^{a}$ ed. São Paulo: Ground; 2009.
9. Kalsa GS. Iridologia integrada: a ciência e a arte da revelação do holograma humano. São Paulo: Mandras; 2009.

10. Battello C. Iridiologia total. São Paulo: Ground; 1996.

11. Salles LF, Silva MJP, Batello CF. A prevalência dos sinais iridológicos que sugerem diabetes nos idosos: um estudo piloto. Cadernos de Naturologia e Terapias Complementares. 2012;1(1):23-8.

12. Squizani BL. Disglicemia e iridologia. [Monografia]. São Paulo (SP): Faculdade de Ciências da Saúde de São Paulo; Centro de Ensino Superior de Homeopatia; 2005.

13. Polit DF, Beck CT, Hungler BP. Fundamentos da Pesquisa em Enfermagem: métodos, avaliação e utilização. Porto Alegre: Artmed; 2006

14. World Health Organization (WHO). Health topics: Obesity. Available from: http://apps.who.int/bmi/index. jsp?introPage=intro 3.html [Acesso em 01 jun. de 2011].

15. US Departament of Health and Human Services. Physical Activity Guidelines for Americans. Available from: http://www.health.gov/ paguidelines [Acesso em 01 jun. de 2011].

16. Lotufo PA. Increasing obesity in Brasil: predicting a new peak of Cardiovascular mortality. Med J Rev Paul Med. 2000;118(6):161-2.

17. Doria A. Genetics of type 2 diabetes. In: Kahn CR, Weir GC, King GL, Jacobson AM, Moses AC, Smith RJ. Joslin's diabetes mellitus. 14th ed. Lippincott Wlliams \& Wilkins; 2005. Chapter 22. p. 371-98.

18. Reis AF, Velho G. Genetic bases of type 2 Diabetes Mellitus. Arq Bras Endocrinol Metab. 2002; 46(4):426-32. 